\title{
FORMACIÓN EN PRÁCTICAS COMUNITARIAS EN LA FACULTAD DE PSICOLOGÍA DE LA UNR. \\ PROBLEMÁTICAS Y ESTRATEGIAS DIDÁCTICAS DESDE LOS ENFOQUES SOCIO-CRÍTICOS EN EDUCACIÓN
}

\author{
Gaspar Manuel Aita \\ Universidad Nacional de Rosario, Argentina \\ superogro@hotmail.com
}

Recibido: 2/07/2020 - Aceptado: 14/12/2020

\section{Resumen}

Desde el Área II: "Prácticas Comunitarias desde la perspectiva psi para la Promoción de la Salud Mental", perteneciente a la asignatura Práctica Profesional Supervisada "A" (Facultad de Psicología de la Universidad Nacional de Rosario), el presente artículo tiene como intención principal reflexionar críticamente en torno a los procesos de formación académica vinculados al campo comunitario desde las perspectivas psi en el nivel de pre-grado.

Es decir, se pretende compartir el estado de situación en que se encuentra este campo en nuestra Facultad y, a partir de ello, pensar posibles estrategias didácticas particulares, posicionados en lo teórico e ideológico desde los enfoques socio-críticos en educación

Desde nuestra experiencia docente, la propuesta emergente está orientada al análisis crítico de aquello que nos acontece dentro de las aulas durante el proceso de enseñanza y aprendizaje para lo comunitario, para habilitarnos a la posibilidad de pensar una didáctica especial y específica, una forma estructurada, sistematizada y planificada, según criterios propios e inherentes al campo de reflexión y acción en las comunidades.

Palabras clave: Prácticas comunitarias desde la perspectiva psi - Formación en el campo comunitario - Estrategias didácticas particulares - Enfoque socio-crítico - Vínculo teoría/práctica.

\section{ACADEMIC BACKGROUND IN COMMUNITY PRACTICES IN THE UNR PSYCHOLOGY SCHOOL. \\ PROBLEMS AND DIDACTIC STRATEGIES FROM THE SOCIO-CRITICAL APPROACHES IN EDUCATION}

\footnotetext{
*Egresado con orgullo de la Universidad Nacional de Rosario en las Carreras de Psicología, Profesorado en Ciencias de la Educación, Licenciado en Ciencias de la Educación y Magister en Literatura para niños y niñas. Actualmente se desempeña como docente en la Facultad de Psicología, en el Área II: Prácticas Comunitarias desde la perspectiva psi para la Promoción de la Salud Mental, perteneciente a la Asignatura Práctica Profesional Supervisada "A". También como docente en el nivel Secundario y como psicólogo del equipo técnico de la Escuela Especial DINAD de Rosario.
} 
ReVista De La Escuela de Ciencias de LA EduCACIÓN, AÑo 17, NRO. 16, VOL. 2, JULIO A DiCIEMBRE dE 2021. PÁGINAS 78-87. ISSN 2362-3349 (EN LÍNEA). FORMACIÓN EN PRÁCTICAS COMUNITARIAS EN LA FACULTAD DE PSICOLOGÍA DE LA UNR. PROBLEMÁTICAS Y ESTRATEGIAS DIDÁCTICAS DESDE LOS ENFOQUES SOCIO-CRÍTICOS EN EDUCACIÓN. GASPAR MANUEL AITA.

\begin{abstract}
From Area II: "Community Practices from the Psychological Perspective for the Promotion of Mental Health", part of the subject Práctica Profesional Supervisada "A" (Supervised Professional Practices A), Psychology School National University of Rosario, the following article has as main intention to critically reflect upon the processes of academic formation, linked to the community field from the psychological perspectives in the level of pre-grade.

It is worth mentioning that, to share the situation in which this area of the university is and, through this, to think about possible personal didactic strategies we should be positioned in the theoretical and ideological level from the socio-critical approaches in education.

From our teaching experience, the emergent proposal is oriented to the critical analysis of that that occurs within the classrooms during the processes of teaching and learning for the communitarian, to allow the possibility of thinking about a especial and specific didactic, a structured form, systematized and planned, according to the proper criteria and inherent reflection and action fields in the communities.
\end{abstract}

Keywords: Community practices from the psychological perspective - Communitarian field formation - Particular didactic strategies - Socio-critical approach - Link theoretical/practical.

\title{
Introducción
}

Aproximadamente desde el año 2017, docentes, alumnos y alumnas del Área II "Prácticas Comunitarias desde la perspectiva psi para la Promoción de la Salud Mental" -perteneciente a la asignatura Práctica Profesional Supervisada "A" (en adelante, PPS "A")-, venimos llevando adelante una serie de investigaciones, encuentros y jornadas que tienen como intención principal el poder reflexionar críticamente en torno a los procesos de formación académica vinculados al campo comunitario, que la Carrera de Psicología de la Universidad Nacional de Rosario (UNR) ofrece a los y las estudiantes en el nivel de pregrado.

En este sentido, el objetivo fundamental que guía este escrito es compartir el estado de situación en que se encuentra el campo comunitario al interior de nuestra carrera. Y, a partir de dicho objetivo, pensar posibles estrategias didácticas particulares destinadas a la formación de los y las estudiantes para el abordaje e intervención en el ámbito comunitario desde las perspectivas psi ${ }^{1}$, posicionados en lo teórico e ideológico desde los enfoques socio-críticos en educación

Habilitarnos para el debate sobre la docencia y sus propuestas en el campo comunitario. Contribuir con la apuesta por interpelar, problematizar acerca de cómo enseñamos para el futuro desempeño en las comunidades. Permitirnos ahondar y desentrañar interrogantes en torno a las maneras en que planificamos, estructuramos y sistematizamos nuestros espacios áulicos. Definir cuáles y cómo serán nuestros roles y tipos de intervenciones. Indagar, reflexionar y propiciar alcanzar instancias de construcción colaborativa de nuevas y específicas propuestas didácticas.

\section{Menciones, apariciones e irrupciones de "lo comunitario" en la Facultad de Psicología de la UNR}

A modo de contextualización para abordar el estado de situación de las prácticas comunitarias al interior de nuestra carrera, en diferentes investigaciones y presentaciones (Aita, 2018) adoptamos el análisis crítico de los Planes de Estudio de 1984 (el primero luego del retorno a la democracia), 1996 (con una serie de modificaciones de suma importancia para este recorrido) y 2014 (el último elaborado para la Carrera de Psicología).

Un período de historia compuesto por 30 años, altamente significativos, que reflejan un proceso histórico "reciente" para la formación académica en el campo de lo comunitario. Puesto que las indefiniciones y yuxtaposiciones conceptuales y de paradigmas en relación a las prácticas comunitarias, aún se manifiestan, emergen y son posibles de ser visibilizadas concretamente en las trayectorias de alumnos y alumnas que eligen formarse en este campo en el último año de la carrera. Como efecto de sus atravesamientos curriculares, subyace en sus inquietudes, dudas y reflexiones, año tras año, en el tramo final de sus formaciones.

Las prácticas comunitarias desde la perspectiva psi, atrapadas en la red discursiva de lo clínico y lo psicoanalítico, han logrado sostenerse entre diversos anudamientos, visualizarse entre disputas históricas y políticas, irrumpir en medio de luchas disciplinares, para luego volver a ocultarse, velarse. En debates sobre si es posible concebirlas como una rama autónoma de la Psicología, si puede o no de convivir con el Psicoanálisis o si "lo comunitario" debe incluirse y depender del macrocosmos que supone "lo social".

La Facultad de Psicología de Rosario, a pesar de las destacables y diversas actividades y propuestas que vienen desarrollando los y las docentes de distintas cátedras desde el primer Plan de Estudios tras el retorno a la democracia en 1984, parece estar en los primeros pasos de la reflexión teórica, ideológica y de

${ }^{1}$ Denominación tentativa, momentánea, que intenta representar e incluir de un modo lo suficientemente abarcativo, los diferentes posicionamientos que en nuestra carrera coexisten en relación a este campo: psicología social, de las instituciones, de las organizaciones, psicoanálisis, teorías de los sistemas, aportes desde la Gestalt, entre otros. 
ReVista De La Escuela de CIENCIAS de LA EduCACIÓN, AÑo 17, NRO. 16, VOL. 2, JULIO A DiCIEMBRE DE 2021. PÁGINAS 78-87. ISSN 2362-3349 (EN LÍNEA). FORMACIÓN EN PRÁCTICAS COMUNITARIAS EN LA FACULTAD DE PSICOLOGÍA DE LA UNR. PROBLEMÁTICAS Y ESTRATEGIAS DIDÁCTICAS DESDE LOS ENFOQUES SOCIO-CRÍTICOS EN EDUCACIÓN. GASPAR MANUEL AITA.

abordajes en el terreno de lo comunitario. Vale decir, está recorriendo los caminos incipientes, iniciando los debates en torno a cómo pensar los vínculos entre estos variados enfoques que actualmente se encuentran abordando y nutriendo -aunque cada uno por su lado- nuestros desarrollos en este campo. Por lo tanto, el desafío amerita ser aceptado. Explorando, rastreando en las raíces históricas, los fragmentos, los modos particulares de existencia, de aparición fugaz o provisoria de las prácticas comunitarias dentro de la Carrera.

En presentaciones anteriores (Aita, 2018) hemos mostrado las primeras aproximaciones a los Planes de Estudio de los años 1984 y 1996, obtenidas a partir de criterios de exploración delimitados (Mención explícita a "lo comunitario"; Alcances y delimitaciones teóricas, metodológicas y de abordaje respecto a otros espacios; Categorías, conceptos o nociones que lo describen, representan o desarrollan; Vínculos con otras teorías, disciplinas o áreas de acción):

Como puede observarse, tras estas primeras lecturas analíticas y críticas respecto a los Planes de Estudio de 1984 y 1996, son bastante escasas las manifestaciones que, desde lo escrito, lo "planeado", hacen referencia directa y específica al campo de lo comunitario.

Al menos hasta aquí, la primera y más concreta la encontramos en aquel punto 9, mencionado en los "Alcances de título" del Plan 1996.

Lo que no es posible dilucidar, por el momento, es desde qué disciplinas, perspectivas teóricas y de abordaje se lo supone (Aita, 2018, p. 15).

Ahora bien, en el año 2011, la Facultad de Psicología de la UNR se presenta por consenso democrático al proceso de Acreditación que propone la Comisión Nacional de Evaluación y Acreditación Universitaria (CONEAU). Decisión que se reafirma al siguiente año:

El 5 de junio del 2012 nuestro Consejo Directivo consolida la posición de acreditar nuestra carrera. Acreditar es reconocernos como institución educativa del Estado, dispuestos a poner en tensión los principios neoliberales de la L.E.S. del '95 y a participar políticamente en la elaboración pública de una Universidad que construya conocimientos con otros, y que reestablezca la relación entre autonomía universitaria y la realidad social².

El Dictamen resultante de los Pares Evaluadores sugiere una serie de requerimientos a implementar. Entre varios señalamientos apuntados, en uno de ellos se señala que: "El plan no contempla todos los Contenidos Curriculares Básicos listados en el Anexo I de la Resolución ME № 343/09 con un tratamiento adecuado y se observa ausencia de contenidos (...)" (CONEAU. Acreditación de Carreras de Psicología y Licenciatura en Psicología. Convocatoria. Informe de Evaluación, 2011, p. 10) Se requiere, además: "Revisar la valoración otorgada a las perspectivas teóricas tratadas a fin de asegurar una formación generalista con pluralismo teórico." (CONEAU. Acreditación de Carreras de Psicología y Licenciatura en Psicología. Convocatoria. Informe de Evaluación, 2011, p. 23)

Un pluralismo que se pone en cuestión a partir de observarse que ciertos contenidos y perspectivas parecen ser abordados en profundidad, mientras que otros no reciben el mismo tratamiento dentro de la formación. Tal es el caso de aquellos contenidos dedicados al Psicoanálisis, los cuales posee más espacios curriculares que otras perspectivas teóricas, con un mayor número de materias y de horas,

Por otro lado, cabe destacar que el eje temático que corresponde al área social y comunitaria, figura como una de las perspectivas un tanto desfavorecidas en su abordaje a lo largo del Plan. Los Pares Evaluadores manifiestan que "no tienen el mismo tratamiento, ya que se ofrecen en seminarios electivos. En este sentido, no se garantiza el dictado de los contenidos correspondientes." Y respecto a la intervención social comunitaria, señalan que, "si bien se pueden observar que las asignaturas Estructura Psicológica Social del Sujeto I, II y III abordan diferentes enfoques de la psicología social, se omite lo comunitario, específicamente modelos y estrategias de intervención en crisis comunitarias." (CONEAU. Acreditación de Carreras de Psicología y Licenciatura en Psicología. Convocatoria. Informe de Evaluación, 2011, p. 10)

\section{Plan de Estudios 2014}

Considerando, entonces, estos y otros requerimientos y observaciones la Facultad de Psicología de la UNR confeccionará su nuevo Plan de Estudios en el año 2014, a través de la Resolución CD № 140/14 y la Resolución CS № 437/14.

Al adentrarnos en la exploración del mismo, encontramos en la Finalidad la propuesta por una capacitación "en las distintas corrientes de pensamiento y escuelas de la Psicología" (Resolución N 140/2014 CD, p. 2). Sólo que, a continuación, se efectúa un agregado, una especie de aclaración, especificación en cuanto al "propósito" de la formación:

(...) profesionales comprometidos con su rol social y los derechos humanos, capaces de intervenir en los problemas psicológicos que se plantean en relación con la salud, el trabajo, el derecho, la

\footnotetext{
${ }^{2}$ (https://fpsico.unr.edu.ar/academica/doc-para-la-acreditacion/).
} 
ReVista De La Escuela de Ciencias de LA EduCACIÓN, AÑo 17, NRO. 16, VOL. 2, JULIO A DiCIEMBRE dE 2021. PÁGINAS 78-87. ISSN 2362-3349 (EN LÍNEA). FORMACIÓN EN PRÁCTICAS COMUNITARIAS EN LA FACULTAD DE PSICOLOGÍA DE LA UNR. PROBLEMÁTICAS Y ESTRATEGIAS DIDÁCTICAS DESDE LOS ENFOQUES SOCIO-CRÍTICOS EN EDUCACIÓN. GASPAR MANUEL AITA.

comunidad y la educación; tanto en el plano individual, grupal o institucional (...) (Resolución № 140/2014 CD, p. 2).

Este agregado podría ser importante en, al menos, dos aspectos. El primer aspecto es que señala el compromiso con el rol social del y de la profesional. Desde el enfoque, por ejemplo, de la Piscología Social Comunitaria es un punto fundamental y el estar señalado aquí podría dar pistas del posicionamiento teórico de este Plan. Y el segundo aspecto es que aparece "la comunidad" como un ámbito explícito y autónomo (sin el agregado "social") de posible intervención, aunque no se realicen especificaciones metodológicas o conceptuales.

A continuación, en los Alcances del Título, aunque son menos que los propuestos en el Plan '96 (16 en lugar de 20), aparecen de forma detallada 3 puntos pertinentes para este escrito:

2. Prescribir y realizar acciones de evaluación, diagnóstico, pronóstico, tratamiento, seguimiento, recuperación y rehabilitación psicológica en los abordajes: individual, grupal, de pareja, familia, instituciones, organizaciones y en lo social-comunitario (Resolución № 140/2014 CD, p. 2).

Vuelve a aparecer esta forma de mención amplia y poco específica a lo "social-comunitario", que nos desorienta en tanto no termina de aclarar si el abordaje sería desde la Psicología Social, la Psicología Comunitaria o la Psicología Social Comunitaria. Pero aun así figura el campo de acción. Resultan significativos, puntualmente, los tipos de intervenciones sugeridas. En cuanto a la evaluación, diagnóstico, pronóstico o seguimiento, pueden ser adecuadas para todas las áreas especificadas. Ahora bien, el tratamiento, recuperación y rehabilitación psicológica resultan tipos de intervenciones un tanto difíciles para pensar en el ámbito de lo comunitario. Estas denominaciones podrían ser resabios de la mirada clínica, psicoanalítica, que, desde hace mucho tiempo, contiene esta Facultad y que fue señalada por la misma CONEAU

11. Diagnosticar, evaluar, asistir, orientar y asesorar en lo concerniente a los aspectos psicológicos en el área social-comunitaria para la promoción y la prevención de la salud y la calidad de vida de grupos y comunidades y la intervención en situaciones críticas de emergencias y catástrofes (Resolución № 140/2014 CD, p. 3).

Este es, quizás, el más importante de los propuestos para este campo. Puesto que se destacan clara y específicamente los "aspectos psicológicos" en relación al área "social-comunitaria" (aunque los términos "social" y "comunitario" continúan unidos en la denominación) No es menor, además, las menciones a la promoción, prevención y la calidad de vida de las comunidades, en tanto podrían trazar algún camino hacia los posicionamientos teóricos desde dónde se suponen estas prácticas. Enlazándose, a la vez, con los lineamientos de la nueva Ley de Salud Mental.

15. Realizar estudios e investigaciones en las diferentes áreas del quehacer disciplinar, a saber: a. Los procesos psicológicos y psicopatológicos a lo largo del ciclo vital. b. Los procesos psicológicos involucrados en el desarrollo y funcionamiento de los grupos, instituciones, organizaciones y la comunidad. c. La construcción y desarrollo de métodos, técnicas e instrumentos de intervención psicológica (Resolución № 140/2014 CD, p. 3).

Parecido al punto anterior en cuanto a la relación con los procesos psicológicos, sólo que ahora se agrega la investigación y eso es destacable. Por otro lado, no es posible dilucidar -como a lo largo de los 3 planes explorados- si "la comunidad" es un término genérico o una categoría. Quizás en esta ocasión sea lo primero, puesto que si no probablemente le hubiesen agregado "lo social".

Continuando con estos análisis, nos abocamos también al apartado que describe el Perfil del Título. Éste se presenta:

(...) formado para la intervención en los ámbitos: clínico, educativo, jurídico forense, social comunitario, organizacional laboral, entre otros. Sus incumbencias son múltiples, desde la evaluación psicológica, asistencia en psicoterapias en lo individual, grupal y familiar, institucional, organizacional y socio-comunitario, planificación de programas de prevención y promoción de la salud e intervención en conflictos y mediación (Resolución № 140/2014 CD, p. 3).

Este punto llama bastante la atención. Nótese cómo en el mismo párrafo, en tanto ámbito se menciona como social comunitario; y en tanto incumbencia como socio-comunitario. Más aun, como incumbencia aparece ligado en la redacción a la "asistencia en psicoterapias...". La presencia de la coma luego de lo "socio-comunitario" parece separarla de la "planificación". ¿Eso quiere decir que se concibe alguna forma de asistencia en psicoterapia socio-comunitaria? De ser así, asomaría nuevamente alguna mirada clínica sobre este campo.

Por último, uno de los aportes quizás más significativos, puede leerse en el punto correspondiente a la Organización del Plan de Estudios. Puesto que allí se destaca la presencia de las 8 áreas que componen este Plan. Las mismas son definidas como "unidades de integración temática, escenario de construcción 
ReVista De La Escuela de CIENCIAS de LA EduCACIÓN, AÑo 17, NRO. 16, VOL. 2, JULIO A DiCIEMBRE DE 2021. PÁGINAS 78-87. ISSN 2362-3349 (EN LÍNEA). FORMACIÓN EN PRÁCTICAS COMUNITARIAS EN LA FACULTAD DE PSICOLOGÍA DE LA UNR. PROBLEMÁTICAS Y ESTRATEGIAS DIDÁCTICAS DESDE LOS ENFOQUES SOCIO-CRÍTICOS EN EDUCACIÓN. GASPAR MANUEL AITA.

de conocimientos y de líneas de investigación en torno a las modificaciones de prácticas y estrategias metodológicas que impliquen la síntesis creativa de teoría y práctica" (Resolución N.1 140/2014 CD, p. 2). La presencia de las mismas es una innovación respecto a los Planes anteriores (1984 y 1996) y se fundamenta en tanto permiten gradualizar e integrar los conocimientos con la realidad social, a partir de un abordaje interdisciplinar y entre las cátedras.

Entre ellas, y aquí se destaca la importancia, aparece en primer lugar el Área Social Comunitaria. Textualmente se expresa lo siguiente:

Integra los aportes y abordajes de la lingüística, la sociología y las teorías de la psicología social, de la psicología comunitaria, los desarrollos teóricos y prácticos de la psicología institucional, de la psicología organizacional, de la psicología en el trabajo y de la salud y la salud mental para la construcción y problematización de lo social cultural entramados en el conocimiento de lo psíquico. Comprende las siguientes asignaturas: Lingüística y Discursividad Social, Teoría Social, Psicología Social y Comunitaria, Organizaciones e Instituciones, Psicología en el Trabajo, Psicología en el Ámbito Jurídico - Forense y Salud Pública y Salud Mental (Resolución N.ํ140/2014 CD, p. 4).

Desde ya que la aparición del Área en sí, es destacable. Más aun, la que aquí se manifiesta podría ser la primera mención a la Psicología Comunitaria en tanto rama, desarrollo o teorización autónoma dentro del campo psi, realizada dentro de un Plan de Estudios de la Carrera de Psicología de la UNR.

Puede observarse también, que dicha Área se encuentra sustentada por asignaturas pertenecientes a distintos años académicos. Lo cual indicaría cierto valor o consideración sistemática, sostenida y multidisciplinaria a lo largo de la formación.

Ahora bien, tras analizar los Planes de Estudio de los años 1984, 1996 y 2014, pareciera que desde lo planeado, escrito, manifiesto y planificado deliberadamente en estos documentos, no se contaría aún con un posicionamiento teórico, ideológico, metodológico y de abordaje lo suficientemente claro en torno a las prácticas comunitarias.

El estado actual de referencia y planificación de estas prácticas, con la saludable incorporación del Área Social Comunitaria en el Plan de 2014, lo encontramos luego y a partir de los requerimientos propuestos por los pares evaluadores de CONEAU. Afirmación que no invalida, desacredita, borra, quita méritos o deja por fuera los trabajos, actividades, seminarios y proyectos de investigación e intervención en los barrios de muchos y muchas docentes. Como así tampoco, resalta o jerarquiza el papel de CONEAU, más allá de su importante rol evaluador.

Tal como expresáramos más arriba, en estas instancias iniciales de debate en torno los posicionamientos y modos de abordaje para este campo particular, emerge como saludable la instauración de la mencionada Área Social Comunitaria en tanto espacio de reflexión, de construcción, creación y visualización de las prácticas comunitarias desde la perspectiva psi en la Facultad de Psicología de la UNR.

\section{La formación académica para el abordaje en las comunidades}

Ahora bien, parte del desafío de estas indagaciones tiene que ver con los modos en que se traduce, se vincula esta historia reciente en los desarrollos en el campo comunitario con las posibles propuestas didácticas orientadas a la formación de nuestros alumnos y alumnas para el abordaje en las comunidades. $\mathrm{O}$, dicho de otro modo, expresado como interrogante, ¿en qué momentos, en qué tramos de todo el proceso que supone nuestra Carrera, formamos específicamente para el ámbito comunitario?

Con más de 15 años de trabajo docente en la mencionada asignatura PPS "A", en cada nuevo ingreso de estudiantes al Área II, en cada inicio de la última etapa de la Carrera de Psicología en UNR, registramos de manera recurrente la emergencia, la incertidumbre que los y las alumnas sienten ante el "desconocimiento" o "falta de información" respecto a este campo.

Aunque no en todos o todas, la sensación que predomina es la de "no saber", "no estar preparados o preparadas", o "saber muy pocas cosas" en relación a ello: qué son las comunidades y qué puede hacer una psicóloga o psicólogo en ellas, desde dónde pensar el trabajo, qué tipo de pedidos podrán hacernos y cómo abordarlos. Estas prácticas les aparecen mezcladas, confundidas con teorías varias o identificadas con autoras y autores "sueltos", descontextualizados. Prácticas ligadas fuertemente a la teoría psicoanalítica y a una mirada clínica "de consultorio" como impronta histórica de la Facultad.

Desde nuestra Área II, tales situaciones, lejos de representarnos una problemática individual de cada alumno o alumna -sea por no haber leído o estudiado los textos "correspondientes", por no cursar con determinado o determinada docente, o sea por no asistir a tal o cual Seminario Electivo-, decidimos interpretarlas desde alguna perspectiva curricular, vinculadas a los Planes de Estudios que definieron y definen los Objetivos de la formación académica. De allí es que surgieron las exploraciones detalladas más arriba.

Más aún, nuestro espacio dentro de la PPS "A" asume desde el año 2020 la denominación "Prácticas comunitarias desde la perspectiva psi para la Promoción de la Salud Mental" también producto de estos interrogantes sobre la historia y el desarrollo de "lo comunitario" al interior de la Carrera.

En este sentido, no perseguimos aquí la búsqueda de definiciones o posicionamientos "originales" en torno a categorías, modos de abordaje o delimitaciones epistemológicas. A partir de la experiencia docente, la propuesta emergente está orientada a la reflexión sobre aquello que nos acontece dentro de las aulas 
ReVista De La Escuela de Ciencias de LA EduCACIÓN, AÑo 17, NRO. 16, VOL. 2, JULIO A DiCIEMBRE dE 2021. PÁGINAS 78-87. ISSN 2362-3349 (EN LÍNEA). FORMACIÓN EN PRÁCTICAS COMUNITARIAS EN LA FACULTAD DE PSICOLOGÍA DE LA UNR. PROBLEMÁTICAS Y ESTRATEGIAS DIDÁCTICAS DESDE LOS ENFOQUES SOCIO-CRÍTICOS EN EDUCACIÓN. GASPAR MANUEL AITA.

durante el proceso de enseñanza y aprendizaje para el campo de lo comunitario. Esto es, la posibilidad de pensar una didáctica especial y específica para este ámbito.

Entendemos a la didáctica como una responsabilidad activa, un trabajo de intervención y -tal como la definiría Edith Litwin (1996)- una "teoría acerca de las prácticas de la enseñanza, significadas en los contextos socio-históricos en que se inscriben" (Camillioni et. al. 1996, p. 94).

Vale decir, un verdadero compromiso de identificación y un posicionamiento ideológico, ético y político, que nos obliga a mirar las formas que tenemos de estrucurar nuestro quehacer docente en el ámbito comunitario: “¿qué enseñamos?, ¿cómo lo enseñamos?, ¿qué debemos enseñar?” (Camillioni et. al. 1996, p. 32)

Un esfuerzo por contextualizarnos, situarnos en relación a nuestra historia, nuestra tradición, nuestra realidad.

Compartimos con José Contreras Domingo (1990) la idea de adoptar un posicionamiento crítico en relación a la Didáctica, que nos permita reflexionar acerca de aquello que estamos produciendo y reproduciendo a partir de nuestras enseñanzas:

(...) es necesario que la Didáctica se guíe por una idea de emancipación y de justicia social que vaya más allá de los fines internos que nos propongamos para los procesos de enseñanza, de tal manera que les dé sentido a esos fines, que los sitúe en el marco general del entramado social en los que tales procesos ocurren (Contreras, 1990, p. 44).

Desde los enfoques socio-críticos en educación, una forma de pensar una posible Didáctica para lo comunitario, en íntima relación con nuestro devenir dentro de la historia de la Carrera de Psicología de la UNR y nuestras circunstancias concretas respecto a las comunidades donde nos desempeñamos. Que trascienda los intereses individuales para dar paso a miradas colectivas que contribuyan a la formación de futuros psicólogas y psicólogos comprometidos socialmente, que colaboren en los procesos de organización y sistematización de las prácticas comunitarias. Y, en este sentido, al retomar a Henry Giroux (1983), Contreras Domingo (1990) nos abre una puerta, un camino para continuar nuestras reflexiones:

(...) podemos crear (...) bolsillos de resistencia que proporcionen modelos pedagógicos para nuevas formas de aprendizaje y de relaciones sociales, formas que puedan usarse en otras esferas más directamente implicadas en la lucha por una nueva moralidad y una nueva visión de la justicia social (Contreras, 1990, p. 37).

Es decir, intentar construcciones cooperativas que quizás no modifiquen totalmente las condiciones históricamente arraigadas en relación a las prácticas docentes para el campo comunitario. Pero sí que permitan interpelaciones institucionales y docentes, que colaboren en transformar y transformarnos, con pequeñas pero sistemáticas estrategias, los modos en que acontecen las actividades áulicas para esta área.

"Bolsillos de resistencia" entendidos como contribuciones en la búsqueda por comprender, repensar, intentar transversalizar nuestras perspectivas y prácticas comunitarias a lo largo de todo el proceso académico. Que posibiliten imaginarnos y situarnos activamente en los actuales contextos nacionales y latinoamericanos. A revisar críticamente las referencias teóricas heredadas, los supuestos que cada alumno y alumna trae en tanto saberes y conocimientos previos.

El propio Henry Giroux (1992) -también desde los enfoques socio-críticos en educación- supone a nuestros espacios académicos insertos en contextos históricos y políticos, que condicionan y determinan nuestras prácticas. Las cuales quedan, por lo tanto, inevitablemente emparentadas al concepto de ideología. Un concepto que nos fuerza a preguntarnos acerca de los intereses políticos y sociales que subyacen a nuestros propios supuestos teóricos y pedagógicos, como paso previo y permanente a la práctica áulica.

\section{Algunas problemáticas en torno a la docencia para la formación comunitaria}

En un breve pero interesantísimo artículo elaborado en 1997 por Fernando Giuliani y Maritza Montero, titulado La docencia en la psicología social comunitaria: algunos problemas, autor y autora se enfocan allí en un aspecto particular de la Psicología Social Comunitaria, inherente a ella, al cual consideran fundamental: "la formación de los psicólogos y psicólogas comunitarios" (Giuliani y Montero, 1997, p. 115).

Desglosan y especifican de forma problemática una serie de instancias que, según ambos escriben, obstaculizarían o dificultarían los procesos de formación académica en el campo comunitario. Instancias que remiten, principalmente, a cuestiones institucionales, propias de la Universidad en la que se desempeñan:

1. Los lapsos académicos y los tiempos comunitarios.

2. La familiarización recíproca.

3. El rol de las/os psicólogas/os.

4. El compromiso.

Con la intención de "mantener una mirada crítica sobre la actividad docente", Giuliani y Montero efectúan una interpretación sumamente interesante: 
ReVista De La Escuela de CIENCIAS de LA EduCACIÓN, AÑo 17, NRO. 16, VOL. 2, JULIO A DiCIEMBRE DE 2021. PÁGINAS 78-87. ISSN 2362-3349 (EN LÍNEA). FORMACIÓN EN PRÁCTICAS COMUNITARIAS EN LA FACULTAD DE PSICOLOGÍA DE LA UNR. PROBLEMÁTICAS Y ESTRATEGIAS DIDÁCTICAS DESDE LOS ENFOQUES SOCIO-CRÍTICOS EN EDUCACIÓN. GASPAR MANUEL AITA.

Quizás uno de los aspectos en los cuales debemos detenemos es que la adquisición de herramientas teóricas y metodológicas a través de un entrenamiento académico no es suficiente para convertir al estudiante exitoso en un psicólogo o psicóloga comunitaria. Ese aprendizaje es sin duda necesario e imprescindible, más no suficiente (Giuliani y Montero, 1997, p. 127).

Una cita valiosa, en tanto nos dejan en claro que la formación de los futuros psicólogos y psicólogas para este campo, no pasa solamente por "la adquisición de herramientas teóricas y metodológicas".

Un poco más adelante, reconocen que uno de los obstáculos que mayormente se les presenta lo constituye "el rol construido en la formación previa que han recibido los estudiantes a lo largo de su carrera" (Giuliani y Montero, 1997, p. 121). Y adecuadamente proponen:

Hemos intentado facilitar esos procesos. Por una parte, a través de los contenidos programáticos de la materia; por otra, propiciando permanentemente el sentido reflexivo de nuestro quehacer, problematizando con los estudiantes nuestra postura epistemológica y nuestras prácticas, a la vez que analizando cada sesión de trabajo, de manera que los estudiantes puedan reconocer los aportes que hacen los miembros de la comunidad, así como su coincidencia y su disidencia respecto de los conocimientos establecidos en la praxis desarrollada hasta ahora (Giuliani y Montero, 1997, p. 122).

Creemos, pues, que de aquí podrían emerger algunas pistas capaces de conducirnos, guiarnos en la búsqueda de herramientas didácticas en la formación académica orientada al trabajo comunitario. Partiendo de procesos de reflexión profunda, confrontando las propias concepciones referidas a las comunidades, problematizando las posturas epistemológicas con las prácticas, analizando cada sesión de trabajo, revisando contenidos específicos de la disciplina.

En este punto y por lo señalado más arriba, los desarrollos de Giuliani y Montero (1997) resultan un aporte relevante y enriquecedor para aquellos y aquellas que intentamos formar académicamente a las futuras generaciones de psicólogos y psicólogas en el campo comunitario. Más aún, si tenemos en cuenta que son muy pocas las investigaciones o artículos publicados, dedicados a la enseñanza específica de esta área.

La relevancia de este pequeño artículo está dada, justamente, por la posibilidad que nos brinda para pensar en que podría existir una didáctica particular abocada a la formación de profesionales psi, que deseen desempeñarse en el área comunitaria. Vale decir, una forma de "enseñanza" estructurada, sistematizada y planificada, según criterios propios e inherentes a esta disciplina o campo de acción. Con propuestas didácticas, actividades áulicas que puedan diferenciarse de aquellas que se ofrecen para otros núcleos disciplinares al interior de la Carrera de Psicología: clínicas psicoanalíticas, investigación, historia y epistemologías, biología y psicodiagnósticos, etc.

Ahora bien, autor y autora sugieren que "es necesario que nos problematicemos nosotros, los psicólogos comunitarios, que hurguemos en nuestras múltiples realidades" (Giuliani y Montero, 1997, p. 126). Hagamos nuestra, entonces, estas intenciones e intentemos reflexionar lo que nos acontece en nuestra Facultad de Psicología de la UNR en relación a la formación para el abordaje comunitario.

Desde el Área II (PPS "A") coincidimos con los planteos anteriormente expuestos como obstáculos por Giuliani y Montero (1997). Y, a partir de nuestra propia experiencia como docentes investigadores e investigadoras, nos animamos a realizar algunos agregados sobre dos de aquellos puntos.

En primer lugar, en referencia al "Rol de las/os psicólogas/os", observamos que la problemática fundamental se plantea a partir de una concepción del o la profesional en tanto "experto" que obstaculizaría o se contrapondría a la "posición epistemológica en que se fundamenta la psicología comunitaria". Una concepción que, autor y autora atribuyen al "rol [profesional] construido en la formación previa que han recibido los estudiantes a lo largo de su carrera" (Giuliani y Montero, 1997, p. 121).

Si pensamos, entonces, los roles o concepciones de los y las profesionales que transmite la Facultad de Psicología de la UNR, nos encontramos también en cierta desventaja respecto al campo comunitario. Desde la elaboración del Plan de Estudios en 1984, se mantiene sobre la práctica y el rol profesional esperado "una formación previa", una orientación de perspectiva clínica. Sustentada, preferentemente, por posicionamientos psicoanalíticos derivados de los planteos freudianos y/o lacanianos en su gran mayoría. Si bien la implementación del Plan de Estudios en 2014 supone posibilidades de apertura y alternativas hacia otros enfoques epistemológicos y tipos de abordaje, como también la oportunidad de profundizar y estimular corrientes o áreas un tanto desatendidas hasta el momento.

Quedaría, sí, por indagar si este tipo de formación redunda o no en la posición de "experto" que autor y autora señalan.

Entonces, cómo afrontaríamos esta fuerte e histórica impronta, esta "formación previa", desde nuestros espacios de trabajo vinculados con lo comunitario. Puesto que, a pesar del tiempo que nos separa de este pequeño artículo que decidimos utilizar como disparador, podemos encontrar ciertas similitudes con nuestra actual situación.

En segundo lugar, nos referiremos a "los lapsos académicos y los tiempos comunitarios": 
ReVista De La Escuela de Ciencias de LA EduCACIÓN, AÑo 17, NRO. 16, VOL. 2, JULIO A DiCIEMBRE dE 2021. PÁGINAS 78-87. ISSN 2362-3349 (EN LÍNEA). FORMACIÓN EN PRÁCTICAS COMUNITARIAS EN LA FACULTAD DE PSICOLOGÍA DE LA UNR. PROBLEMÁTICAS Y ESTRATEGIAS DIDÁCTICAS DESDE LOS ENFOQUES SOCIO-CRÍTICOS EN EDUCACIÓN. GASPAR MANUEL AITA.

Es éste, a nuestro juicio, uno de los aspectos que dificultan la labor comunitaria orientada desde la academia. Impartimos un curso cuya duración es de un semestre académico, lo cual, en tiempo de acción directa, no supera los cuatro meses «calendario». De ese tiempo efectivo, nuestro programa toma alrededor de un mes para una primera fase de contenidos teóricos, en la cual se intentan cubrir los supuestos epistemológicos de la disciplina, sus principales marcos conceptuales y los aspectos éticos de su aplicación; aspectos éstos que constituyen un mínimo de conocimientos básicos necesarios antes de incorporar a los estudiantes al trabajo comunitario. A partir de esta fase se inicia la etapa de familiarización, con lo cual el tiempo de trabajo comunitario se reduce entonces a dos o tres meses (Giuliani y Montero, 1997, p. 117).

Si nos circunscribimos a los plazos con que contamos en nuestra Área II, las coincidencias son casi totales en cuanto al obstáculo académico que supone la escasez del "tiempo de acción directa" para el trabajo. Tanto con los y las estudiantes, como con las comunidades.

Ahora bien, vale señalar que aún con estas dificultades, contamos con cierta ventaja metodológica aunque bien podríamos decir pedagógica-. Puesto que es uno de los únicos espacios curriculares -de hecho, en el campo de lo comunitario es el único- en donde las alumnas y alumnos tienen contacto directo con las comunidades y recién acceden al mismo en el último año de la Carrera.

Por lo tanto, entonces, estaríamos en condiciones adecuadas para plantear como posible interrogante qué sucede, cómo se aborda didácticamente el ámbito comunitario en las asignaturas de los años académicos anteriores, que no cuentan con estas instancias y vínculos con las comunidades.

Volviendo al trabajo de Giuliani y Montero (1997), de aquella cita vinculada a los lapsos académicos y los tiempos comunitarios, otra cuestión es importante para destacar en un intento por aproximarnos a esta problemática.

En dicha cita se menciona que, antes de iniciar la etapa de familiarización y como parte inherente al proceso de enseñanza que describen, destinan "alrededor de un mes para una primera fase de contenidos teóricos" -referidos a la PSC, por supuesto- para el abordaje de aspectos que "constituyen un mínimo de conocimientos básicos necesarios antes de incorporar a los estudiantes al trabajo comunitario" (Giuliani y Montero, 1997, s/p)

¿Por qué consideramos esto importante, digno de resaltarse? ¿Qué aspectos continúan invitándonos a la reflexión a pesar de la diferencia temporal que nos separa de aquel escrito? ¿Por qué suponemos que puede aclararnos en algo la situación en nuestra Carrera, respecto a aquellos alumnos y alumnas que decidan formarse para el ejercicio comunitario?

Quizás sea porque pone en evidencia la concepción de enseñanza que poseemos, mediante la cual primero debemos impartir "contenidos", "conocimientos" teóricos, para luego poder realizar las prácticas.

Y tanto es así, que en aquella revisión del Plan 2014, con su división en Áreas, nos encontramos con esta concepción que parece operar a contramano de los supuestos que plantea la Psicología Social Comunitaria. Porque dentro del Área Social Comunitaria sólo observamos asignaturas "teóricas" -en las cuales es muy posible que los y las docentes se esfuercen por modificar y transformar- pertenecientes a los 5 primeros años de la Carrera. Y porque sí existe un Área de las Prácticas pero que contiene una sola asignatura, de 6to. año, que es ni más ni menos que la PPS en donde se encuentra incluida nuestra Área II. Vale decir, nosotros también concebimos como necesario atravesar 5 años académicos, con 6 asignaturas "teóricas", antes de poder ingresar a los efectores a realizar las prácticas, los abordajes, las aproximaciones a las comunidades.

Cómo y desde dónde repensar esta supuesta "separación" entre la teoría y la práctica en el quehacer docente, en el acontecer áulico será, entonces, una de las problemáticas fundamentales que podríamos dejar formulada. Vale decir, cómo, desde y entre quiénes podemos ser capaces de encontrar las pistas necesarias, considerando nuestras actuales condiciones de cursado -según figura en el Plan 2014- para el campo comunitario.

\section{¿Hacia una didáctica para la formación comunitaria?}

Cada propuesta de enseñanza y aprendizaje lleva implícita, no sólo una concepción de educación, sino también una forma determinada de concebir a los y las estudiantes -qué esperamos de ellos y ellas y cuáles serán nuestros roles como formadores y formadoras-, junto a un posicionamiento ético y político en cuanto a lo social y los modos de participación colaborativa que podrían asumir las comunidades.

Por lo tanto, suscribir esta búsqueda de posibles estrategias didácticas específicas para el campo comunitario desde los enfoques y teorías críticas en educación, podría ser una forma de trazar una adecuada relación ideológica y epistemológica con los supuestos de la Psicología Social Comunitaria -en el caso nuestro serían, aún, las prácticas comunitarias desde la perspectiva psi- Un proyecto que podría ser extensible a todas las asignaturas que conforman el ASC, incluso las que no contemplen salidas o prácticas en terreno como el caso de la PPS.

Según lo desarrolla Peter McLaren (1984), estos posicionamientos críticos permiten incorporar los hechos sociales, históricos y políticos, a la interpretación de las prácticas docentes. Nos proporciona, además, "una tensión dialéctica entre teoría, práctica y experiencia, una tensión necesaria para el aprendizaje crítico. (McLaren, 1984, p. 253)

Tal como lo describe el autor: 
ReVista De La Escuela de CIENCIAS de LA EduCACIÓN, AÑo 17, NRO. 16, VOL. 2, JULIO A DiCIEMBRE DE 2021. PÁGINAS 78-87. ISSN 2362-3349 (EN LÍNEA). FORMACIÓN EN PRÁCTICAS COMUNITARIAS EN LA FACULTAD DE PSICOLOGÍA DE LA UNR. PROBLEMÁTICAS Y ESTRATEGIAS DIDÁCTICAS DESDE LOS ENFOQUES SOCIO-CRÍTICOS EN EDUCACIÓN. GASPAR MANUEL AITA.

La pedagogía crítica se pregunta cómo y por qué el conocimiento es construido en la forma en que lo hace, y cómo y por qué algunas construcciones de la realidad son legitimadas y celebradas por la cultura dominante mientras que otras no lo son. La pedagogía crítica se pregunta cómo nuestro conocimiento de sentido común cotidiano -nuestras construcciones sociales o "subjetividades"- es producido y vivido. En otras palabras, ¿cuáles son las funciones sociales del conocimiento? El factor crucial aquí es que algunas formas de conocimiento tienen más poder y legitimidad que otras (McLaren, 1984, p. 268).

Siguiendo la línea que plantean Wilfred Carr y Stephen Kemmis (1988), consideramos que esta reflexión sobre el vínculo teoría y práctica podría ser una vía posible -aunque podría haber otras- para iniciar procesos de análisis y escritura que contribuyan a la búsqueda didáctica antes mencionada.

Si bien no recorreremos en esta ocasión las argumentaciones de ambos autores en favor de la investigación-acción, sí nos abocaremos a algunas cuestiones en relación al vínculo teoría y práctica desde el enfoque socio-crítico que proponen. Un vínculo que conciben como un proceso dialéctico contextualizado, de reflexión y lucha política, orientado hacia la propia emancipación y al "mejoramiento de las condiciones sociales y materiales bajo las cuales tiene lugar la práctica" (Carr y Kemmis, 1988, p. 158).

Un posicionamiento que, llevado al plano pedagógico, se muestra sumamente adecuado para interactuar con los enfoques que componen la Psicología Social Comunitaria.

Y en relación a lo analizado anteriormente en relación al Plan de Estudios 2014 de la Facultad de Psicología de la UNR, siguiendo lo que se plantea desde estos enfoques críticos, lo teórico no podría quedar escindido de lo práctico, dividido por Áreas, asignaturas o años académicos. Estableciendo, determinando de antemano que primero debe acontecer una cierta cantidad de "teoría", para luego poder acceder a una "buena instancia práctica".

Ahora bien, que ambos -teoría y práctica- puedan interactuar dialécticamente, también es una decisión, una voluntad, parte de un proceso de autorreflexión crítica que podríamos proponernos los y las docentes. Al menos quienes estamos incluidos e incluidas en el ASC. Vale decir, la relación teoría/práctica en el plano de lo comunitario debería revisarse, estructurarse, planificarse y transformarse en actividades áulicas específicas, quizás más que en otras áreas.

Coincidimos con Carr y Kemmis (1988) en pensar que todas las teorías son producto de alguna actividad práctica y que, a su vez, toda actividad práctica recibe orientación de la teoría de una u otra forma. Y si adoptamos esta relación en tanto proceso dialéctico y político, en tanto revisión ideológica y epistemológica, podríamos aproximarnos a algunas alternativas -y no estaría mal pensarlas en términos didácticos- a la "separación" entre teoría y práctica establecida en nuestro Plan 2014 para la formación comunitaria. Donde aquellos conceptos o categorías "fundamentales" puedan entrar en juego, dialécticamente y desde los inicios de nuestra formación, con aquellas comunidades y con cada estudiante y docente en tanto portadores de historias, saberes y conocimientos. Donde no sean "transmitidas" exclusivamente por medio de un cúmulo de autores y autoras - de suma relevancia, desde ya- en su mayoría provenientes de contextos socio-históricos y políticos lejanos a las comunidades en donde alumnos y alumnas pretenderán desarrollar sus futuras prácticas.

Por el contrario, intentar contactos, acercamientos, a esas comunidades -que no dejan de ser nuestras comunidades- para que puedan cimentarse desde los inicios de la formación académica los primeros trazos, las primeras experiencias de aquello que implica el trabajar colaborativamente, cuerpo a cuerpo junto a otros y otras, el afrontar injusticias y desigualdades para la búsqueda de alternativas. Incentivar concepciones respecto a nuestros roles en la sociedad a partir de perspectivas inclusivas, que sean permeables a sentir, a vivenciar y enfrentar los actos de discriminación y violencia contra los grupos más vulnerados en sus derechos fundamentales. Que no les resuenen como hechos lejanos, ajenos, la pobreza a que está sometida gran parte de nuestra población. Más aún, que puedan percibir que es allí donde está el campo de acción y transformación de nuestra práctica, el lugar en donde más necesitan nuestra capacidad de reflexión y actuación conjunta.

Parafraseando y retomando los planteos de Henry Giroux (1992), será nuestra tarea diseñar dispositivos pedagógicos que sean significativos para los y las estudiantes, que sean críticos respecto a la realidad universitaria y que sean transformadores en tanto herramientas de cambio para las comunidades. Revisar y reflexionar en torno a quiénes somos como docentes, cómo seleccionamos los contenidos a tratar y de qué manera los ofrecemos para que encuentren resonancia en las experiencias vitales de los y las estudiantes. Qué vínculos establecemos con otros campos disciplinares.

De la mano de esta primera discusión sobre la teoría y la práctica, abrimos los interrogantes en torno a qué estrategias didácticas estamos utilizando para interpelar y reflexionar cada tema escogido con aquello que nos sucede, tanto en nuestra realidad inmediata, nacional, como en los contextos latinoamericanos en que estamos insertos.

Tal como proponían Giuliani y Montero (1997), la apuesta es "la problematización constante; es el intento permanente por romper viejos moldes" (Giuliani y Montero, 1997, p. 125). En un proceso que "se construye y reconstruye en el trabajo y en la reflexión crítica" (Giuliani y Montero, 1997, p. 126). Y la exigencia, entonces, es problematizarnos de manera permanente nosotros y nosotras en tanto docentes del Área Comunitaria. 
ReVista De La Escuela de Ciencias de LA EduCACIÓN, AÑo 17, NRO. 16, VOL. 2, JULIO A DiCIEMBRE dE 2021. PÁGINAS 78-87. ISSN 2362-3349 (EN LÍNEA). FORMACIÓN EN PRÁCTICAS COMUNITARIAS EN LA FACULTAD DE PSICOLOGÍA DE LA UNR. PROBLEMÁTICAS Y ESTRATEGIAS DIDÁCTICAS DESDE LOS ENFOQUES SOCIO-CRÍTICOS EN EDUCACIÓN. GASPAR MANUEL AITA.

\section{Referencias bibliográficas}

Aita, G. (2018). "Practicas Comunitarias en la Facultad de Psicología de la UNR: Debates y procesos de constitución". Presentado en: XI Congreso Iberoamericano de Psicología y XVII Congreso Argentino de Psicología: La profesión en su laberinto: teorías, prácticas, territorios. (11 al 13 de octubre, 2018) Córdoba. Argentina.

Camillonl, A. et. al. (1996). Corrientes didácticas contemporáneas. Buenos Aires: Paidós.

Carr, W. y Kemmis, S. (1988). Teoría crítica de la enseñanza. La investigación-acción en la formación del profesorado. Barcelona: Ediciones Martínez Roca, S. A.

Giroux, H. (1992). Teoría y resistencia en educación. México - Argentina: Siglo XXI editores.

Giuliani, F. y Montero, M. (1997). La docencia en la psicología social comunitaria: Algunos problemas. Psykhe, 8, 114127.

Litwin, E. (1996). El campo de la didáctica: en busca de una nueva agenda. En: Camillioni, A. et. al. (1996). Corrientes didácticas contemporáneas. Buenos Aires: Paidós.

McLaren, P. (1984). La vida en las escuelas. Una introducción a la pedagogía crítica en los fundamentos de la educación. Argentina: Siglo XXI Editores.

Resolución Consejo Directivo N.ำ 140/2014 CD (2014) Universidad Nacional de Rosario. 